\title{
LA SUPERACION DOCENTE CONTINUA: ALGUNOS CRITERIOS PARA SU PERFECCIONAMIENTO
}

\author{
Nemecio Núñez R. y Pedro G. Palacios C. \\ Universidad Católica Santo Toribio de Mogrovejo, Perú
}

\section{INTRODUCCIÓN}

Hasta finales del siglo XIX se consideraba que los profesionales se formaban para toda la vida, en ese tiempo el desarrollo de la ciencia era lento y más aún la introducción de sus adelantos, era la época del telégrafo, del correo por cartas, la comunicación entre los profesionales era insegura y asincrónica, se enviaba una carta y tardaba tres meses en llegar y luego quien la contestaba tardaba otros tres meses, seis meses para comunicarse y recibir la contestación o la opinión, no tenían ni remotamente la inmediatez que tienen hoy las nuevas técnicas de la informática con las cuales las ideas se desarrollan y difunden a otra velocidad.

La ciencia de la educación no escapaba a esta situación. Durante siglos, se consideró que la calidad de la enseñanza se lograba a través de la enseñanza enciclopédica por el profesor, y el papel de éste era, ante todo, llenar de conocimientos la mente de los alumnos, como si se tratase de un recipiente vacío. No se consideraba el enseñar a hacer y enseñar a aprender. Las únicas habilidades importantes eran aquellas que se relacionaban con la memorización y repetición de voluminosos textos, si se lograba eso, entonces se consideraba cumplida la función de la educación (UNESCO - Informe D’ Lors: 1996).

En esa época, los métodos de aprendizaje se fundamentaban en que los estudiantes aprendían básicamente a partir de escuchar a un erudito profesor, a través del dictado de un curso, y las habilidades que más necesitaba el alumno eran las de escuchar y ver.

Los conocimientos en las instituciones docentes se daban para toda la vida y realmente ningún profesional sentía necesidad de cualificarse de nuevo durante el transcurso de su desempeño profesional, si lo hacía era a partir del auto estudio.

Por eso, los profesionales de la educación no sentían la necesidad de una acción de superación y, por el contrario, se sentían ofendidos si alguien les hubiese propuesto recibir un curso de capacitación, un postgrado, etc. Un perfeccionamiento profesional que ahora nos parece muy necesario y deseable.

\section{SITUACIÓN ACTUAL}

Estamos a principios del siglo XXI. Muchas cosas en nuestra sociedad han variado, la ciencia se desarrolla vertiginosamente y las aplicaciones de sus resultados se realizan en el transcurso de pocos años y aun de menos tiempo.

Las comunicaciones han sobrepasado todo lo inimaginable. No somos capaces de emplear eficientemente todo lo que nos brinda y mucho menos de analizar todo el volumen de información que sobre una sola área del conocimiento se acumula -digamos - en Internet. 
Ya la información, al igual que otros campos, se ha globalizado, se ha hecho patrimonio de una parte de la humanidad, pues aún queda otra parte que demorará años en darse cuenta de que ésta existe. (Pedró y Rolo:1998)

Todo ello se debe a que estamos en un mundo globalizado. Desde este punto de vista no estamos aislados, lo que nos obliga a movernos al ritmo de todo el mundo en correspondencia con estándares internacionales, a riesgo de quedarnos atrasados.

Esto es valido también para la educación; ella se ha visto obligada a cambiar sus paradigmas. La enseñanza abandona sus ropajes tradicionales para aplicar nuevos métodos en los cuales los alumnos son considerados sujetos del aprendizaje y no sólo meros objetos.

Ya la educación no es ni mucho menos para toda la vida, la concepción del aprendizaje ante todo es aprender a aprender, dotar de habilidades y capacidades que lo preparen para aprender durante toda la vida, ha surgido el concepto de capacitación continua. (Álvarez de Zayas, C: 1998).

En este momento, los objetivos de la educación no pueden ser ya los mismos. Los nuevos objetivos deberán plantearse según la realidad presente, la cual demanda ante todo, desarrollar la capacidad del individuo al cambio constante. Esto implica que la educación no será ya más la transmisora de la cultura del pasado, sino el instrumento que prepare al individuo para los requerimientos del futuro. (Álvarez de Zayas, C: 1999).

En segundo término, la educación deberá enseñar al individuo cómo acceder a los nuevos centros de información, así como a buscar eficientemente esta información, debido a la extraordinaria acumulación de conocimientos que ha hecho necesaria la habilidad especial de navegar en el mar de información que nos rodea.

Evidentemente, la educación actual requiere de un profesor completamente distinto al de décadas atrás, lo único que ha de permanecer constante es su amor por la profesión.

Cualquier profesor puede explicar y argumentar lo anteriormente expresado incluso en una forma mejor. Sin embargo, en el caso de la educación, por lo menos en el ámbito de América Latina, el discurso y la práctica van distanciados.

\section{REALIDAD DE LA EDUCACIÓN LATINOAMERICANA}

Aún contamos con modelos educativos tradicionales que no han escapado al siglo antepasado, basados en la transmisión de conocimientos memorísticos y enciclopédicos, y a no enseñar a hacer, ni a ser. (UNESCO - Informe D' Lors: 1996).

Ante todo se necesita cambiar el concepto subyacente de que el profesor es el centro del proceso y situar en ese centro al alumno, pero no por declaración sino de hecho. Una muestra de ello es que aún cuando las nuevas concepciones didácticas proclaman la amplia utilización de métodos participativos, nos encontramos con objetivos de los programas elaborados desde el punto de vista de lo que tiene que hacer el profesor y no lo que tiene que alcanzar el alumno, se continúan empleando las clases expositivas y se utiliza poco el trabajo en equipo, no escapa a esa situación el sistema de evaluación obsoleto que aún se practica y que contribuye a desarrollar el memorismo, la repetición de los contenidos y el trabajo individual. (Álvarez de Zayas C.:1999). 
En la actualidad, la educación atraviesa por un mal momento del cual no se sabe cuándo ni cómo despertará. Cada día en las encuestas e investigaciones realizadas por organismos internacionales sobre la educación y el aprendizaje les otorgan los últimos lugares a nuestros países, especialmente, en lo que se refiere a la comprensión lectora, al desarrollo del pensamiento lógico - matemático, la educación en valores; asimismo, crece más el número de analfabetos sin posibilidades de salir de esta situación. (PREAL: 2001).

La grave situación en que se encuentra la educación en nuestros países ha obligado a tomar medidas, a veces de emergencia para tratar de resolver el problema. Sin embargo, en ocasiones, esto ha quedado en el plano formal, sin llegar a incidir en los problemas esenciales de la educación. Pues aún no nos hemos dado cuenta que el concepto mismo de educación va más allá del circunstancial cumplimiento de las funciones de enseñar, crear y difundir el conocimiento, y tiene que ver con los fines, es decir, con los cómo, por qué, y para qué de estas funciones. (Adine, Fátima: 2000).

De lo que se trata entonces es de formar y capacitar al profesor con otra visión de su misión y proporcionarle herramientas más adecuadas a la inmensa tarea que tiene que acometer.

\section{UNA ALTERNATIVA DE SOLUCIÓN}

Consecuentemente, los procesos de cambio e innovación en la educación habrán de concebirse no como recurso circunstancial, sino como tarea permanente e imprescindible.

Se impone una nueva concepción de formar y capacitar a los profesores: primero el profesor no se puede formar para toda la vida, al igual que ningún otro profesional; segundo, esto implica una capacitación continua que incluya un sistema de educación de postgrado encaminado hacia un fin definido previamente sobre la base de las necesidades existentes. (Núñez y Palacios: 2002).

En el Perú, surgió y se aplicó un sistema de capacitación docente desde 1996, el Plan Nacional de Capacitación Docente (PLANCAD) cuyo objetivo declarado es que: "EI PLANCAD ofrece a los docentes de Educación Primaria y secundaria la oportunidad de actualizar sus conocimientos en relación con el Nuevo Enfoque Pedagógico Constructivista, para analizar y poner en práctica nuevas metodologías de trabajo activo, participativo e integrado y el sistema de evaluación coherente con este planteamiento". (Ministerio de Educación: 2001)

No obstante, en la educación peruana estos objetivos persiste una fuerte problemática en la preparación de los profesores que, a nuestro criterio, atribuimos a los programas de capacitación de docentes en servicio en el Perú que se realizan según diferentes metodologías caracterizadas por una fuerte base de empirismo, lo que le resta eficacia y eficiencia al proceso de capacitación.

No estamos propugnando ni mucho menos que desaparezca la capacitación que oficialmente el Ministerio de Educación ofrece a los profesores, pues sabemos lo que ha logrado, aunque no estamos conforme en su totalidad. Muestra de ello es la cantidad de investigaciones sobre este tema que, como tesis de maestrías y doctorados, se defienden actualmente sobre las modificaciones a este plan de capacitación.

De tal manera, frente a la situación actual, surge la necesidad de hacer realidad una nueva manera de concebir y realizar la capacitación de los docentes en servicio, entendida ésta como inherente al desarrollo de su práctica docente. Por tanto, no está sujeta a soluciones de situaciones desarticuladas, sino que, muy por el contrario, aborda el problema desde una visión integradora y holística para formar una 
nueva actitud docente, fundamentada en el dominio de la pedagogía, la didáctica y la investigación, las que le asignan al profesor idoneidad y autoridad en el dominio de la ciencia, del saber enseñar y del saber orientar la formación de sí mismo y de los estudiantes para la transformación de las múltiples realidades en los contextos donde desarrolla su práctica pedagógica.

Nosotros vemos como una posible solución elaborar un programa de capacitación docente continua tal, que involucre armónicamente los contenidos, la investigación educativa, la práctica de la profesión, así como los ejes entendidos como proceso de investigación, interdisciplinariedad, trabajo en equipo, reflexión sobre la práctica.

Pero todo ello articulado y teniendo como centro la formación de las competencias necesarias al profesor, a partir de las cuales se ha de estructurar todo el programa. Entonces, el problema está en determinar cuáles son estas competencias que hace falta formar en el profesor.

Desde ese punto de vista, el programa deberá tener ejes que atraviesen todas sus asignaturas, áreas, disciplinas o módulos. Dichos ejes deben estar formados por disciplinas integradoras que bajo el concepto del logro de una determinada competencia aglutinan conocimientos y acciones de diversos tipos.

El programa de capacitación, además, debe estructurarse sobre la base de los tres componentes clásicos de cualquier currículo (Álvarez de Zayas: 1999), los cuales en una proporción adecuada promueven la formación de un profesor equilibrado en su actuación. Estos componentes son:

- El componente académico: contiene el fundamento teórico necesario a los objetivos del programa y básicamente está contenido en las asignaturas, áreas, disciplinas o módulos.

- El componente laboral: abarca toda la actividad práctica a realizar, pero no la propia de cada asignatura, áreas, disciplinas o módulos, sino aquella concebida como un sistema enfocado hacia alcanzar las competencias requeridas. Está íntimamente relacionada con su actividad diaria como profesor, pero desde el punto de vista de cómo deben hacerse las cosas.

- El componente investigativo: abarca la actividad del profesor como consumidor de los resultados de la investigación realizada por otros, es decir, el profesor capacitado para aplicar los adelantos de la ciencia de la educación.

La actividad del profesor como investigador individual o como parte de un colectivo de profesores investigadores, tratando de determinar la solución a los problemas que enfrenta en su quehacer diario. (Stenhouse, L: 1984).

La actividad del profesor en la enseñanza a sus alumnos da los primeros pasos en la investigación, desde "descubrir" la naturaleza en adelante.

Es necesario dejar bien claro que no estamos promoviendo el énfasis en la práctica, sacrificando o restando importancia a la teoría ni mucho menos orientando hacia la práctica la formación inicial de un profesor, porque volveríamos a las recetas. Lo que queremos es transformar la enseñanza-aprendizaje en una actividad realista y concreta, estructurada con un criterio funcional, más flexible que los enfoques tradicionales (práctica sin teoría o teoría sin práctica) y comenzar a entender esa relación recíproca entre teoría y práctica que se establece en cada toma de decisiones que el profesor realiza en el aula. 
Tanto la actividad laboral como la de investigación deben estructurarse como disciplinas integradoras, las que con un objetivo central transcurren durante todo el tiempo en que dura el programa de capacitación, y en el caso de la actividad investigadora no necesariamente tiene que contar con un volumen de horas, pues puede estar integrada como una idea y con objetivos únicos en cada una de las asignaturas, áreas, disciplinas o módulos. que compongan el programa.

Pero además, el programa de capacitación debe contar con ejes dinamizadores, integradores y orientadores del programa de capacitación docente continua, tales como la interdisciplinariedad, el trabajo en equipo, la investigación y la reflexión sobre la práctica del profesor. (Núñez y Palacios: 2002).

En resumen, estamos tratando de formar al profesor capaz de valerse de la investigación y realizarla, de promover en sus alumnos el espíritu de la inquietud, de la investigación, que posea en su base una fuerte y necesaria teoría pedagógica y en la capacidad de ir autopreparándose a medida que lo requiera, refrendado todo ello por un entrenamiento en la práctica.

\section{OTROS ASPECTOS IMPORTANTES DE LA CAPACITACIÓN DE LOS PROFESORES}

Hay varios aspectos importantes en la concepción de la capacitación de los profesores que es necesario tener en cuenta para un programa efectivo:

a) Concebir a la capacitación docente como un proceso continuo, lo que implica entenderla como inherente al ejercicio profesional de los profesores. (Ornelas, Carlos: 1997)

Esto significa que el profesor debe asumir una dinámica de superación profesional continua, puesto que las tendencias de cambio en el campo científico-tecnológico y, específicamente, pedagógico y didáctico son muy acelerados, por lo que los sistemas educativos están en la imperiosa necesidad de contar con un profesional capacitado para llevar adelante las innovaciones, así como afrontar decididamente estos cambios; ello implica estructurar un sistema de acciones de postgrado. Cuando hablamos de un sistema, no hablamos de acciones aisladas, sin relación, sino de un conjunto de acciones en las cuales las de menor categoría puedan ser acreditadas en las de mayor. Por ejemplo, si un maestro recibe dos o tres cursos reconocidos o acreditados de 3 créditos, estos puedan ser acreditados, además, recibir un entrenamiento, con la finalidad de que pueda convertirse en un diplomado y así sucesivamente en maestrías, etc.

No se trata de pensar en la capacitación como una herramienta externa dirigida a remediar los males -reales o eventuales - de un sistema o para corregir prácticas inadecuadas; no se trata de pensar en la capacitación como un instrumento o una estrategia transitoria que se desactiva una vez que se han cumplido tales popósitos remediables o correctivos. Por el contrario, estamos convencidos de que, en contextos vertiginosos como los que caracterizan a nuestro presente $y$, probablemente a nuestro futuro, la formación docente inicial o de grado es sólo un punto de partida.

En síntesis, el diseño de capacitación que estamos delineando está abierto hacia el futuro, ya que aspira a que los profesores tomen conciencia de la necesidad y de la importancia de la capacitación continua, y la asuman como proceso constitutivo de su quehacer laboral. 
b) La capacitación docente como proceso interdisciplinario.

En los últimos años, la interdisciplinariedad de las ciencias es un tema que en el campo educativo se viene profundizando; ya la creación cultural humana no se desarrolla de manera disciplinaria (Tunnerman, Carlos: 2001). Sin que las disciplinas hayan perdido su vigencia, lo cierto es que las necesidades, los intereses, las tendencias, etc. propiciaron nuevos horizontes en el avance científico. Casi todas las ciencias necesitan de otras para avanzar, a partir de lo cual los paradigmas, los enfoques han variado. Estamos al frente de la interdisciplinariedad, la multidisciplinariedad y la transdisciplinariedad.

c) La capacitación docente como proceso del trabajo en equipo.

La cooperación es un proceso mediante el cual los individuos se apoyan mutuamente, comparten sus tareas y se organizan en torno a un objetivo común. La cooperación crea mejores condiciones de trabajo y avance $y$, por tanto, es de indiscutible beneficio para el aprendizaje y el desarrollo de los individuos.

Nunca hubo tanta necesidad de dominar el trabajo en equipo en las organizaciones sociales tales como la escuela. El profesor tiene que aprender a utilizarla.

\section{CONCLUSIONES}

En el modelo que estamos propugnando para una capacitación continua, en medio de una cultura en movilidad, de una sociedad que exige a la escuela, de una familia en crisis y de una gran confusión de valores, el profesor sigue formándose y aprendiendo mientras enseña. Su "práctica" será el campo donde la formación se transformará en continua.

Al mismo tiempo, pensamos que puede ser conveniente salir de nuestro trabajo solitario, compartir con otros profesores la investigación sobre la propia experiencia. Aceptar que otros miren el producto o el proceso del trabajo en el aula; trabajar cooperativamente en pequeños proyectos. Todo esto ayudará a reflexionar sobre la realidad, a compartir la investigación bibliográfica y a lograr innovaciones que apunten a cambios reales en la escuela.

Por otra parte, en tanto la capacitación es un proceso educativo, debe llevarse a cabo de acuerdo con los mismos principios que sustentan los procesos de enseñanza y de aprendizaje en las escuelas. Aunque con una didáctica que si bien no puede considerarse particular, sí posee características un tanto diferentes. Hay que recordar que la capacitación es para adultos, aunque debe constituirse en un modelo válido de lo que se espera que ocurra en las aulas.

Un último aspecto es el referido a las "modas" en la pedagogía y la didáctica. Pues, en ocasiones, pasa un poco como con los niños y adolescentes que están aprendiendo la informática que siempre quieren tener el último programa, el mejor, el último computador, aunque no sean capaces de sacarles el máximo provecho a lo que poseen en la actualidad.

La situación de la ligereza con la cual se pasa de una teoría pedagógica a otra, con la rapidez que se adopta oficialmente tal o cual modalidad, sòlo por el hecho de que está recomendada por alguien o porque en el país vecino se refieren algunos resultados, es verdaderamente peligroso, pues nos puede 
hacer perder nuestra identidad y, en realidad, lo que pasa es que esos cambios no llegan naturalmente al profesor, sino por la vía administrativa que, en definitiva, cumple sólo cuando recibe la inspección.

Según nuestro criterio, no se trata de desechar las nuevas teorías pedagógicas, sino dejarlas asentar, realizar experimentos de aplicación y, sobre la base de sus resultados, introducirlas en la práctica educativa con características propias. El problema se refiere a que los errores en la educación se pagan a la vuelta de los años, cuando ya no tienen retroceso y se convierten en irremediables.

Al final de la reflexión acerca de la superación continua docente, como siempre, quedan más dudas que certezas. La transición de una concepción a otra será dolorosa, tomará tiempo y tal vez las resistencias serán mayores a las que uno pudiera imaginar, pero seguro que lo lograremos.

\section{REFERENCIAS BIBLIOGRÁFICAS}

ADINE, Fátima y otros.. Diseño Curricular. Habana - Cuba . Edic. Instituto Pedagógico Latinoamericano. 2000.

Álvarez de Zayas, Carlos M. Pedagogía como ciencia. Habana - Cuba. Edit. Universidad de La Habana. 1998.

ÁlvaREZ DE ZAYAS, Carlos M. Diseño curricular. Cochabamba. Bolivia. 1999

DELORS, Jackes y otros. La educación encierra un tesoro, Madrid - España, Ediciones UNESCO. 1996.

Escuela de POSTGRADO DE LA UNPRG. Investigaciones en pedagogía. Primera promoción de doctorado en ciencias de la educación. Lambayeque. Perú. Edit. Universidad Nacional Pedro Ruiz Gallo FACHSE. 2002.

GIMENO SACRISTÁN, J. Poderes inestables en educación. Madrid - España . Editorial Morata. 1998.

MARTINEZ BONAFÉ, Jaume. El profesor como investigador. España. Cuadernos de pedagogía No 160, junio. 1988.

MINISTERIO de EdUCACIÓN DEL PeRÚ. Manual de Capacitación Docente. Lima - Perú. Edit. MED. 2001.

Núnez RojAs, Nemecio y Palacios CONTRERAS, Pedro Gonzalo. Modelo teórico para un programa de capacitación continua de docentes en servicio en educación básica. Tesis presentada en opción al grado científico de doctor en ciencias de la educación. Lambayeque. Perú. 2002.

ORNELAS, Carlos. El perfil del maestro del siglo XXI. Notas para una investigación. Simposio internacional: Educación para el siglo XXI. México. www.secyd-dgo.gob.mx/simposium/ponencias/tema04.txt. 1997

PEDRÓ, Francesc y ROLO, José Manuel. Los sistemas educativos iberoamericanos en el contexto de la globalización. Interrogantes y oportunidades. VIII Conferencia Iberoamericana de Educación, Portugal. 1998

PREAL. Quedándonos atrás. Un informe del progreso educativo en América Latina, Publicaciones PREAL. 2001.

STENHOUSE, L. Investigación y desarrollo del Currículo. Madrid - España. Ediciones Morata. Cuarta edición. 1984 
TUNNERMAN, Carlos. La educación en el horizonte del siglo XXI. Iresalc-UNESCO. Caracas. 2001.

VALDÉS AVALOS, Isabel. Niveles de congruencia entre las prácticas evaluativas realizadas por los docentes y la concepción del aprendizaje que dichas prácticas generan en el estudiante. México . En: spou@orca.ens.cetys.mx,. 1996 


\title{
Contactar
}

Revista lberoamericana de Educación

\author{
Principal OEI
}

